Supporting Information for

\title{
Label-free Isolation and mRNA Detection of \\ Circulating Tumor Cells from Patients with metastatic lung cancer for disease diagnosis and monitoring therapeutic efficacy
}

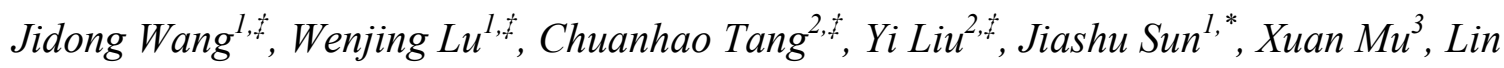

Zhang $^{3}$, Bo Dai ${ }^{4}$, Xiaoyan Li $^{2}$, Hailong Zhuo ${ }^{2}$, and Xingyu Jiang ${ }^{1}$

${ }^{1}$ Beijing Engineering Research Center for BioNanotechnology \& CAS Key Laboratory

for Biological Effects of Nanomaterials and Nanosafety, National Center for Nanoscience and Technology, Beijing, 100190, China.

${ }^{2}$ Affiliated Hospital of Academy of Military Medical Sciences 100071, China.

${ }^{3}$ Peking Union Medical College and Chinese Academy of Medical Sciences, Beijing, 100730, China.

${ }^{4}$ Department of Urology, Fudan University Shanghai Cancer Center, Shanghai, 200032, China.

E-mail: sunjs@,nanoctr.cn

$\$$ These authors contributed equally.

Device fabrication, sample preparation, erythrocyte lysis, LAMP of CK-19 mRNA and Figures S1-S6 are in Supporting Information. 


\section{Device Fabrication}

The SU-8 (MicroChem Corp) mold of double spiral microchannel is fabricated on a 4inch silicon substrate according to the standard soft lithography protocol. Degassed PDMS (mixed in a 10:1 ratio of PDMS base to curing agent, Sylgard 184, Dow Corning Inc.) is cast over the mold, and baked at $100{ }^{\circ} \mathrm{C}$ for $15 \mathrm{~min}$ on a heating plate. The cured PDMS with embedded double spiral microchannel is peeled off from the mold, and the inlet port of the microchannel is punched through the PDMS layer using a flat-tipped needle. The plastic tube coated with a small amount of adhesive sealant (Dow Corning® 3145 RTV) is carefully inserted into the inlet port, and degassed PDMS is poured over the surface of microfluidic chip. The whole chip is baked inside an over at $80{ }^{\circ} \mathrm{C}$ for 45 min to cure the top PDMS layer and to secure the interconnection between plastic tube and inlet port. The cured microfluidic device is diced by razor blade, and three outlet ports are punched through the PDMS using a 2-mm-diameter puncher (Harris). This microfluidic cell sorter is used for separation of large CTCs from lysed whole blood, in which CTCs are converged and deflected to the middle outlet, and white blood cells (WBCs) are focused and out from the inner outlet.

To collect and enrich the separated CTCs, a small piece ( $3 \mathrm{~mm} \times 3 \mathrm{~mm}$ square) of membrane filter is cut out from the polycarbonate track-etch membrane filter of uniform pore size of $8 \mu \mathrm{m}$ in diameter (Millipore Ireland ltd). The small flat filter is glued on the surface of middle outlet with a tiny amount of adhesive sealant (Dow Corning® 3145 RTV). The whole device is baked at $80{ }^{\circ} \mathrm{C}$ for $60 \mathrm{~min}$ in an oven. The assembled microfluidic chip with integrated membrane filter is shown in Figure. S1.

\section{Sample preparation}

A549 cells are detached from culture plates with trypsin/EDTA, washed in DMEM, and centrifuged at $300 \mathrm{rpm}$ for $3 \mathrm{~min}$ to prepare the cell suspension. We use a surfacemodified hydrophobic glass capillary connected with a disposable pipette-tip (Microman CP-25) to siphon suspended A549 cells. The confined A549 cells inside the capillary could be counted under a microscope. After cell counting, we gently push the piston of 
pipette-tip to eject the A549 cells into the healthy blood sample. The spiked A 549 cells range from tens to a hundred cells $/ \mathrm{mL}$ whole blood. To spike a large number of A549 cells into blood, we first count the cells with a hemocytometer, and dilute the cell suspension serially to a desired concentration.

To visualize the cell trajectories inside the microfluidic cell sorter, A549 cells are stained with $\mathrm{C} 34551$ ( $0.5 \%$, orange) for about $1 \mathrm{~h}$ before spiking into the blood. The WBCs are stained with C3099 (0.5\%, green). The whole blood containing A549 cells are treated by two different methods before cell separation: (1) The blood sample is diluted by $30 \times$ using a $0.9 \%$ saline solution; (2) The blood sample is lysed using RBCs lysis buffer (Qiagen) at a ratio of $1 \mathrm{ml}$ blood to $5 \mathrm{ml}$ buffer for $10 \mathrm{~min}$ with gently shaking, and the lysed blood becomes semi-transparent (Figure 2).

\section{Erythrocyte lysis}

$1 \mathrm{ml}$ of peripheral whole blood general contains approximately five billion of RBCs, which may significantly affect the cell trajectories inside the microfluidic cell sorter. In our previous work, we dilute the blood by 20-50 folds, and enable an efficient CTCs separation from diluted RBCs. The issue of blood dilution lies in the dramatically prolonged processing time due to the increased sample volume. The residual RBCs after cell separation may also affect the following immunofluorescence staining and the RNA extraction process. To simplify the CTCs separation and the subsequent CTCs detection from whole blood, the pretreatment of RBCs before cell separation may provide a possible solution. In comparison with the density-gradient centrifugation involving complicated operations to remove the RBCs from whole blood, the direct RBCs lysis is straightforward, one-step and maximally avoids the loss of CTCs in whole blood. Compared with the karyotes such as WBCs and CTCs, the RBCs are more susceptible to hypotonic shock and will burst rapidly in the hypotonic lysis buffer. To demonstrate the efficiency of RBCs lysis buffer, we spike several fluorescently-stained A549 cells into the whole blood, and lyse the RBCs for $10 \mathrm{~min}$. The lysed whole blood is observed under the confocal microscope, showing that the lysis buffer can only burst RBCs while 
maintaining tumor cells intact (Figure S3). The volume ratio of lysis buffer to whole blood is 5, and the lysed blood sample is directly injected into the microfluidic device without other pretreatment.

\section{LAMP of CK-19 mRNA}

A $2 \mu \mathrm{L}$ total RNA sample is added to $23 \mu \mathrm{L}$ of solution consisting of $1 \mu \mathrm{M}$ each of the forward inner (CK19FIP) and reverse primer (CK19BIP), $0.2 \mu \mathrm{M}$ each of forward outer (CK19F3) and reverse primer (CK19R3), $0.8 \mu \mathrm{M}$ each of forward loop (CK19LF) and reverse primer (CK19LR). $0.4 \mathrm{mM}$ deoxynucleotide triphosphates, $20 \mathrm{mM}$ Tris-HCl, 10 $\mathrm{mM} \mathrm{KCl}, 10 \mathrm{mM}(\mathrm{NH} 4)_{2} \mathrm{SO}_{4}, 6 \mathrm{mM} \mathrm{MgSO}_{4}, 0.1 \%$ Triton X-100, $1 \mathrm{M}$ beatine (B0300, Sigma), $1 \times$ SYBR green (Solarbio), 8 units Bst DNA Polymerse (M0275, New England Biolabs), 5 units avian myeloblastosis virus reverse transcriptase (M0277, New England Biolabs) and 25 units RNasin Plus RNase Inhibitor (N2611, Promega). The DNA fragments synthesized by LAMP reaction are detected based of the fluorescence emitted by the dsDNA binded to SYBR green dye. Temperature control for the LAMP reaction and fluorescence intensity measurement are performed in eppendorf Mastercycler. 
Table S1: LAMP primers for CK-19 mRNA

\begin{tabular}{|l|l|}
\hline Primer & \multicolumn{1}{|c|}{ Sequence } \\
\hline FIP-ck19 & $\begin{array}{l}\text { GGA GTT CTC AAT GGT GGC ACC AAC TAC TAC } \\
\text { ACG ACC ATC CA }\end{array}$ \\
\hline BIP-ck19 & $\begin{array}{l}\text { GTC CTG CAG ATC GAC AAC GCC TCC GTC TCA } \\
\text { AAC TTG GTT CG }\end{array}$ \\
\hline F3-ck19 & TGG TAC CAG AAG CAG GGG \\
\hline B3-ck19 & GTT GAT GTC GGC CTC CAC G \\
\hline LF-ck19 & AGA ATC TTG TCC CGC AGG \\
\hline LB-ck19 & CGT CTG GCT GCA GAT GA \\
\hline
\end{tabular}

Abbreviations: F3/B3: outer primer; LF/LB: loop primer; FIP/BIP: inner primer 


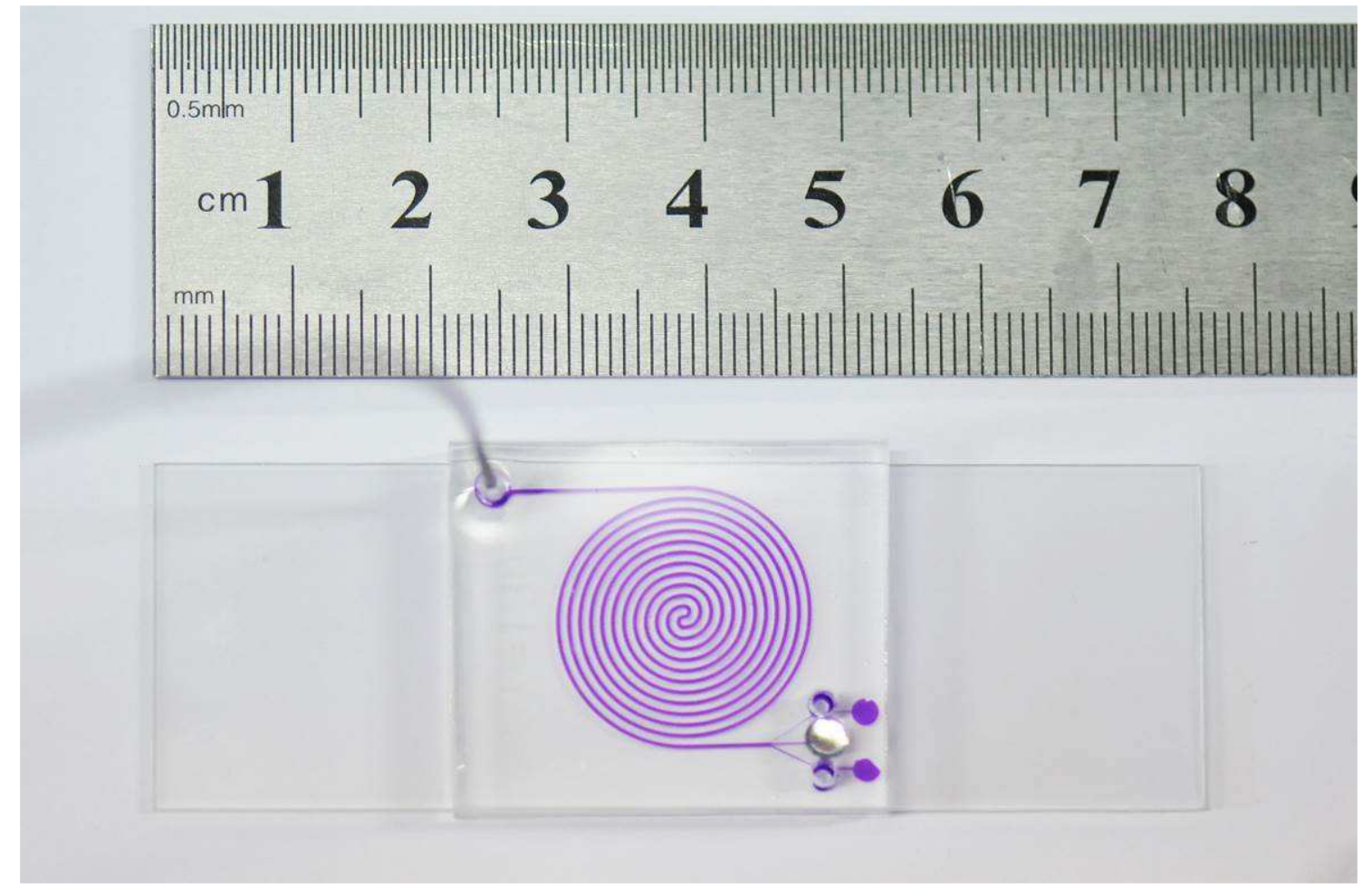

Figure S1. Photography of the inertial-based microfluidic cell sorter combined with an integrated membrane filter. 


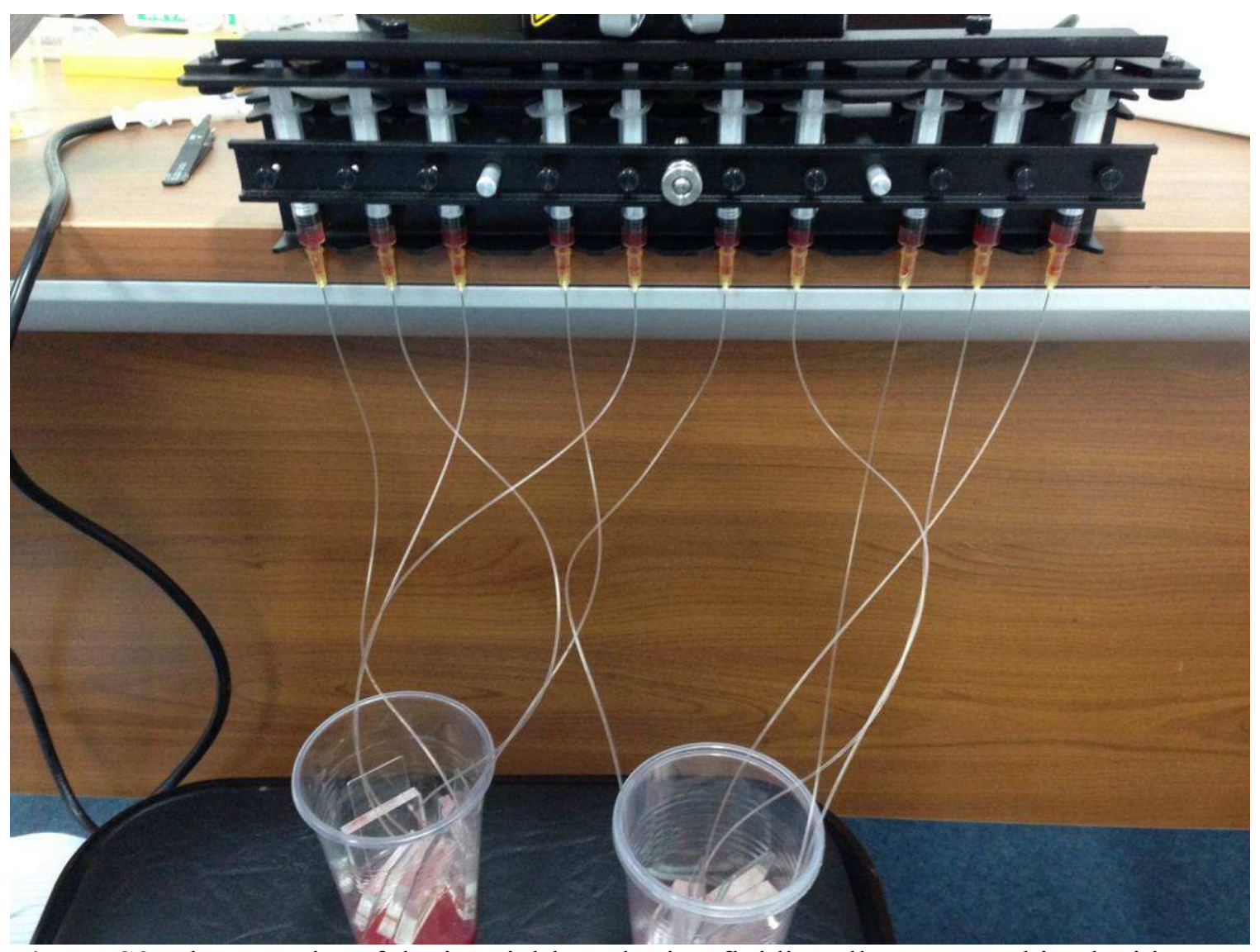

Figure S2. Photography of the inertial-based microfluidic cell sorter combined with an integrated membrane filter. 

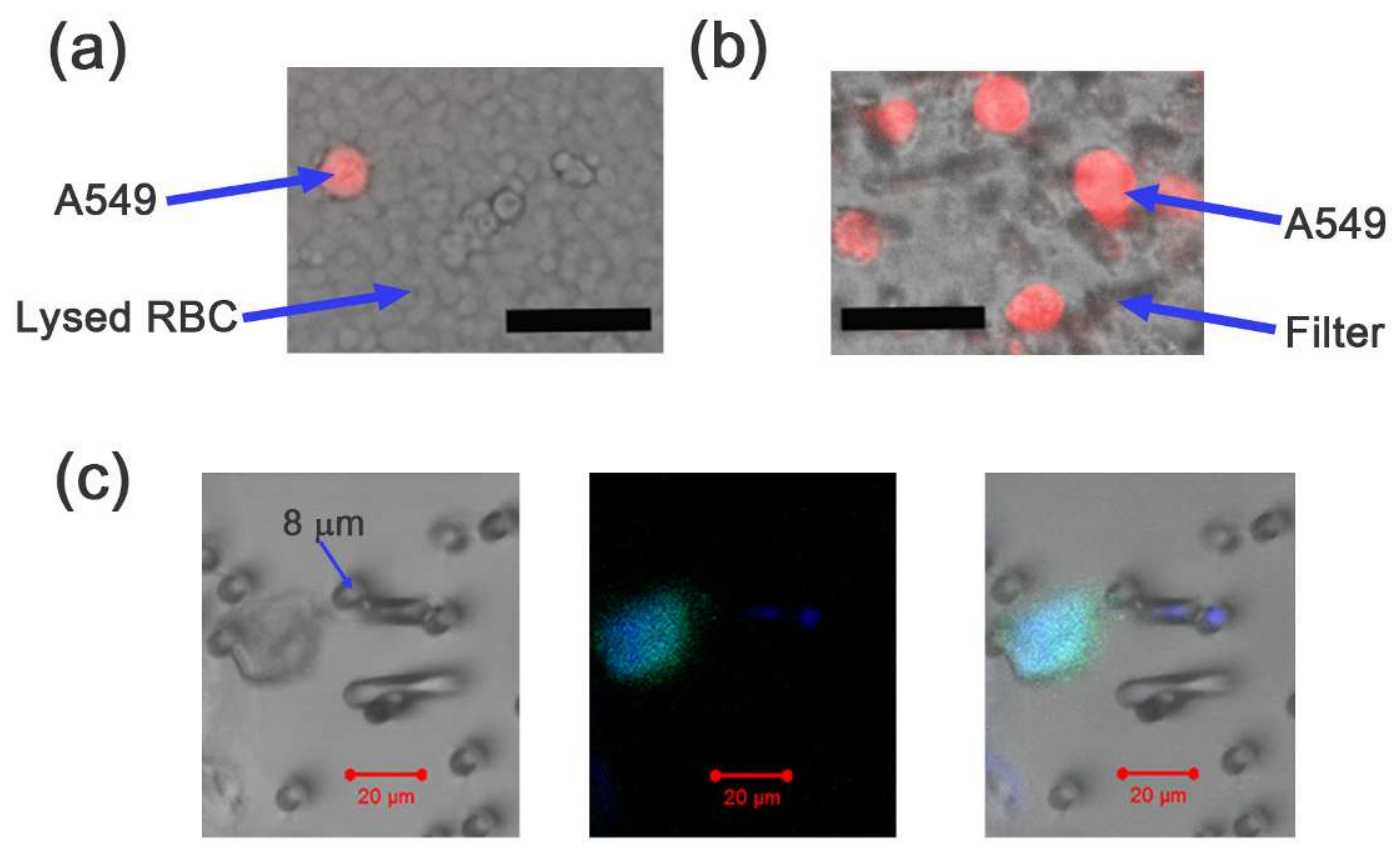

Figure S3. (a) The confocal microscopic image of RBCs-lysed whole blood containing spiked fluorescently-labeled A549 cells. (b) The confocal microscopic image of separated A549 cells that are captured and enriched by membrane filter of pore size of $8 \mu \mathrm{m}$. The scale bar is $50 \mu \mathrm{m}$. (c) The confocal microscopic images of immunofluorescently stained CTCs attached on the membrane filter after cell separation by microfluidic device. 



Figure S4. Microscopic images of separated A549 cells by microfluidic device and normal A549 cells (Control) cultured over a period of $24 \mathrm{hr}$ (a) and $48 \mathrm{hr}$ (b). The scale bar is $20 \mu \mathrm{m}$. 

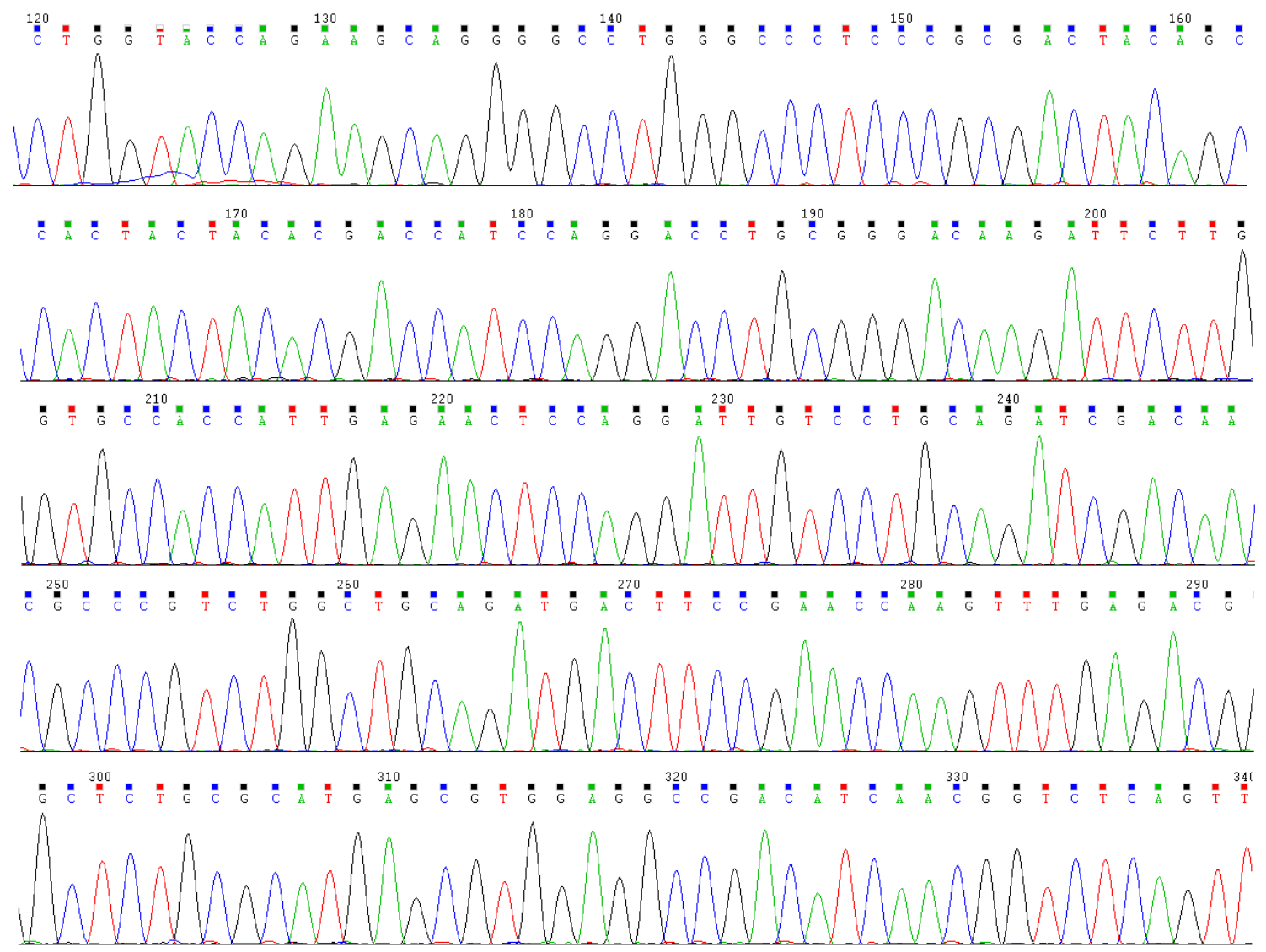

Figure S5. The detailed sequences of CK-19. 


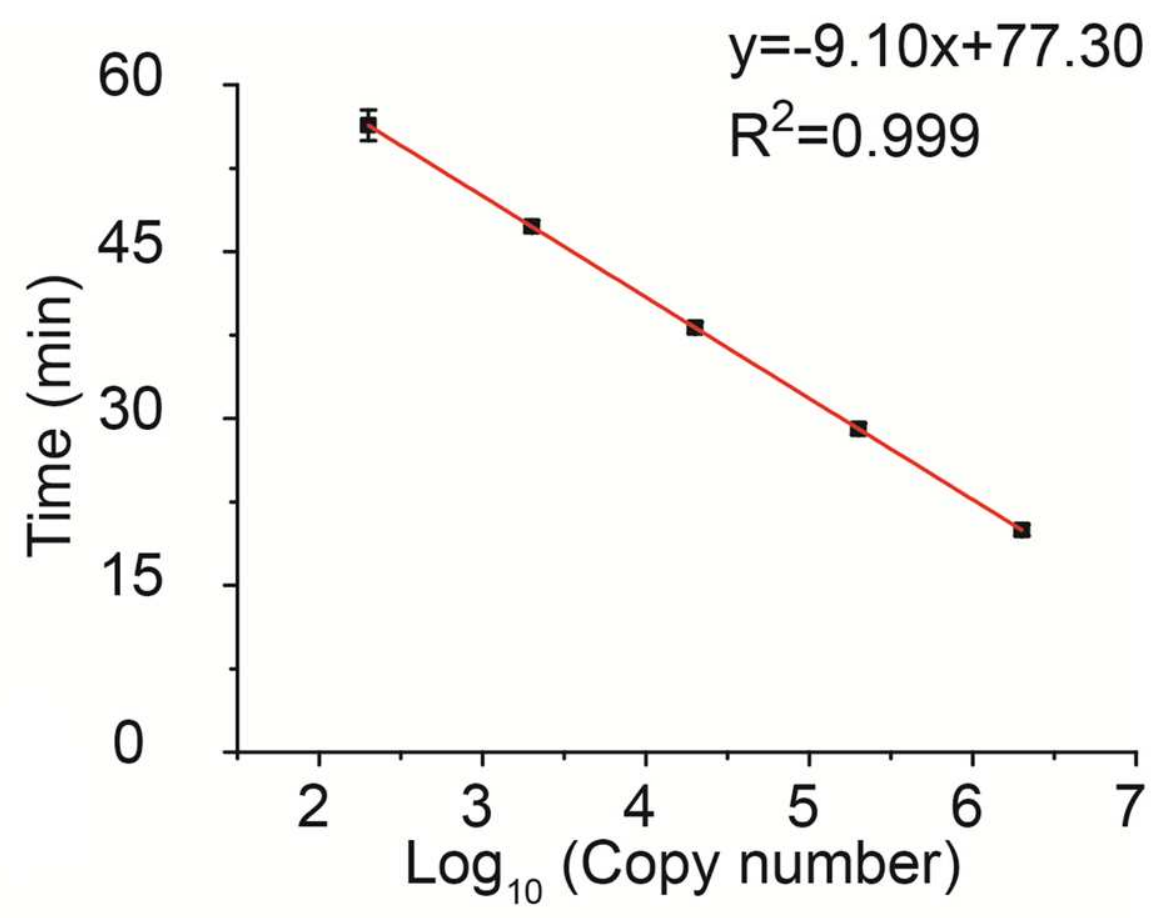

Figure S6. The amplification time of plasmids containing target CK-19 versus the concentrations of plasmids. 